\title{
Heavy-tailed densities
}

Javier Rojo*

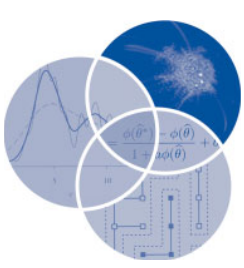

The concept of heavy- or long-tailed densities (or distributions) has attracted much well-deserved attention in the literature. A quick search in Google using the keywords long-tailed statistics retrieves almost 12 million items. The concept has become a pillar of the theory of extremes, and through its connection with outlier-prone distributions, long-tailed distributions also play a central role in the theory of robustness.

The concept of tail heaviness is by now ubiquitous, appearing in a diverse set of disciplines that includes: economics, communications, atmospheric sciences, climate modeling, social sciences, physics, modeling of complex systems, etc. Nevertheless, the precise meaning of 'long-' or 'heavy tails' remains somewhat elusive. Thus, in a substantial portion of the early literature, long-tailedness meant that the underlying distribution was capable of producing anomalous observations in the sense that they were 'too far' from the main body of observations. Implicit in these informal definitions was the notion that any distribution that behaved that way had to do so because its tails were longer than those of the normal distribution. This paper discusses tail orderings and several approaches for the classification of probability distributions according to tail heaviness. It is concluded that an approach based on the limiting behavior of the residual life function, and its corresponding characterizations based on functions of regular variation and asymptotic distribution of extreme spacings, provides the more natural and illuminating concepts of tail behavior. @ 2013 Wiley Periodicals, Inc.

How to cite this article:

WIREs Comput Stat 2013, 5:30-40. doi: 10.1002/wics.1236

Keywords: extreme value theory; functions of regular variation; spacings; failure rate; quantile function

\section{INTRODUCTION}

$\mathrm{T}$ The concept of tail heaviness of a probability distribution has played an important role, and has attracted much well-deserved attention, in several disciplines. For example, Wang and Yatracos ${ }^{1}$ proposed an index to measure right-tail risk that fits well with the pure-tail ordering in $\mathrm{Rojo}^{2}$. The concept of tail heaviness has proven useful in the discussion of outliers and therefore in the area of robustness. The discussion on outliers seems to have originated with astronomical data. As early as 1777, D. Bernoulli ${ }^{3}$ considered the possibility of discarding observations. The following passage from the English translation of Bernoulli's work provides a glimpse of

*Correspondence to: jrojo@rice.edu

Statistics Department, Rice University, Houston, TX, USA
Bernoulli's thinking on rejecting observations deemed to be unusual ${ }^{4}$ :

But is it right to hold that the several observations are of the same weight or moment, or equally prone to any and every error? Are errors of some degrees as easy to make as others of as many minutes? Is there everywhere the same probability? Such an assertion would be quite absurd, which is undoubtedly the reason why astronomers prefer to reject completely observations which they judge to be too wide of the truth, while retaining the rest and, indeed, assigning to them the same reliability. This practice makes it more than clear that they are far from assigning the same validity to each of the observations they have made, for they reject some in their entirety, while in the case of others they not only retain them all but, moreover, treat them alike. I see no way of drawing a dividing line between those that are to be utterly 
rejected and those that are to be wholly retained; it may even happen that the rejected observation is the one that would have supplied the best correction to the others. Nevertheless, I do not condemn in every case the principle of rejecting one or other of the observations, indeed I approve it, whenever in the course of observation an accident occurs which in itself raises an immediate scruple in the mind of the observer, before he has considered the event and compared it with the other observations. If there is no such reason for dissatisfaction I think each and every observation should be admitted whatever its quality, as long as the observer is conscious that he has taken every care.

Legendre, ${ }^{5}$ like Bernoulli, allowed for the possibility of discarding outliers and Peirce ${ }^{6}$ first addressed the issue of what to do in such situations by proposing a criterion for rejecting outlying observations. After Peirce's efforts, there followed several works on the topic of tests for rejecting outliers. Some works, e.g., Refs 7 and 8, were critical of Peirce's proposal. For an interesting account on these early efforts to address the issue of outliers see Ref 9 . Decisions to classify observations as outliers need to be made with reference to an underlying probability model. Thus, an observation may seem to be an outlier when the underlying model is the standard normal distribution, but may not be so if the reference model is the lognormal distribution. Similar considerations led to the development of models for 'heavy-tailed' distributions. These developments included models based on mixtures (convex combinations) of normal densities such as those proposed by Newcomb ${ }^{10,11}$ and which later resurfaced with Tukey. ${ }^{12}$ See, e.g., Ref 13 for an account on the origin of these contamination models and the history of robustness from 1885 to 1920.

Despite the close connection in the literature between the concepts of outliers and heavy-tailed distributions, the precise meaning of the latter remains somewhat elusive. This work aims at providing a precise definition of heavy-tailedness of a distribution. Because of the close connection between heavy-tailedness and outliers, this paper starts with a review of the concepts of outlier-resistant and outlier-prone probability distributions proposed by Neyman and $\mathrm{Scott}^{14}$ and further discussed by Green, ${ }^{15,16}$ Gather, ${ }^{17-19}$ and Mathar. ${ }^{20}$ The literature contains several references to orderings of probability distributions by tail behavior but these orderings are, for the most part, influenced by other aspects of the probability distribution besides tail heaviness. It is possible to define partial orders of distributions based on pure-tail behavior. For this purpose, the work by $\mathrm{Rojo}^{2,21}$ is reviewed and seen to provide a framework for comparing distributions based solely on tail heaviness. Several approaches are considered for classifying probability distributions based on tail behavior. The start is Pareto's work in 1897 that provided a model for heavy-tailed distributions. Pareto's law, motivated by empirical evidence, modeled income by a function of regular variation. The works of Parzen, ${ }^{22}$ Schuster, ${ }^{23}$ and Rojo $^{24}$ are reviewed and it is argued that a definition of heavy-tailedness based on the asymptotic behavior of the residual life function and its corresponding characterizations in terms of functions of regular variation and asymptotic theory of extreme spacings provides the most satisfactory and natural concept.

\section{OUTLIER-PRONE AND OUTLIER-RESISTANT DISTRIBUTIONS}

After observing that most of the methods for identifying outlying observations did so with reference to the normal distribution, Neyman and $\mathrm{Scott}^{14}$ provided guidance in deciding when it was, and it was not, appropriate to reject an 'outlier' without assuming a specific reference model. They proposed the concepts of k-outlier and probability distribution families that are outlier-resistant or outlier-prone.

Hereinafter, let $X_{1}, \ldots, X_{n}$ represent a random sample from a distribution $F$, and let $X_{(1)}, \ldots, X_{(n)}$ denote the corresponding order statistics.

Definition 1. Given $X_{1}, \ldots, X_{n}$ and $k>0, X_{(n)}$ is said to be a k-outlier when

$$
X_{(n)}-X_{(n-1)}>k\left(X_{(n-1)}-X_{(1)}\right) .
$$

The quantity on the left side of Eq. (1) has been termed the extreme spacing in the literature. Denoting by $X_{(i+1)}-X_{(i)}$ the $i$ th data spacing, for $i=2,3, \ldots, n-1$, then $X_{(n)}$ is a k-outlier when the extreme spacing exceeds a k-multiple of the sum of all the other spacings.

Denote by $P_{k, n}(F)$ the probability that a random sample from $F$ of size $n$ contains a k-outlier, and consider a family of probability distributions $\mathcal{F}$, and let $\Pi_{k, n}(\mathcal{F})=\sup _{F \in \mathcal{F}} P_{k, n}(F)$.

Definition 2. A family of distributions $\mathcal{F}$ is said to be $(k, n)$-outlier-resistant when $\Pi_{k, n}(\mathcal{F})<1$. Otherwise, the family of distributions $\mathcal{F}$ is said to be $(k, n)$-outlierprone.

If in addition, the family of distributions $\mathcal{F}$ is $(k, n)$-outlier-prone for all $k>0$ and $n>2$, then $\mathcal{F}$ is said to be outlier-prone completely.

It turns out that if $\mathcal{F}$ is a location-scale family, then the family $\mathcal{F}$ is outlier-resistant. Thus the 
family of normal distributions is outlier-resistant as is, surprisingly, the family $\mathcal{C}(\mu, \sigma)$ of Cauchy distributions symmetric about $\mu$ and with scale parameter $\sigma$. By contrast, the families of lognormal and gamma distributions are outlier-prone completely. Because the lognormal distribution is considered to be long-tailed, the fact that the family is outlierprone is not surprising. On the other hand, it will be seen that the gamma distributions are medium-tailed and, according to the Neyman-Scott definition, they are also outlier-prone completely. These observations suggest that the connection between outlier proneness, or resistance, and tail heaviness of the family of distributions is not captured well through the concepts espoused in the above definitions.

As the above concepts apply to families of distributions, rather than to individual distributions, Green ${ }^{16}$ proposed similar concepts as those proposed by Neyman and Scott but applicable to individual distributions. Hereinafter, let the extreme spacing be denoted by $S_{n}=X_{(n)}-X_{(n-1)}$, the survival function by $\bar{F}(x)=1-F(x)$, and let $T_{n}=\frac{X_{(n)}}{X_{(n-1)}}$. Let $R_{x}(t)=$ $\frac{\bar{F}(t+x)}{\bar{F}(x)}$ denote the residual life function at time $x$. Convergence in probability will be denoted by $\stackrel{P}{\rightarrow}$ and convergence with probability one will be denoted by $\stackrel{\text { a.s. }}{\rightarrow}$. The following definitions are from Ref 16 .

Definition 3. Suppose that $F(\infty)=1$ with $F(x)<1$ for all $x$. Then

(a) $F$ is absolutely outlier-resistant when $S_{n} \stackrel{P}{\rightarrow} 0$.

(b) $F$ is relatively outlier-resistant when $T_{n} \stackrel{P}{\rightarrow} 1$.

(c) $F$ is absolutely outlier-prone when there are constants $M>0, \varepsilon>0$, and $n_{0}$, such that $P\left\{S_{n}>M\right\} \geq \varepsilon$ for all $n>n_{0}$.

(d) $F$ is relatively outlier-prone if there are constants $M>0, \varepsilon>0$, and $n_{0}$, such that $P\left\{T_{n}-1>M\right\} \geq \varepsilon$ for all $n>n_{0}$.

Note that $\underset{P}{(\mathrm{c})}$ and (d) imply that $S_{n} \stackrel{P}{\stackrel{\rightarrow}{0} 0}$ and that $T_{n}-1 \stackrel{P}{\nrightarrow} 0$, respectively. On the other hand, the definition does not consider the cases where, although $S_{n} \stackrel{P}{\nrightarrow} 0$ and $T_{n}-1 \stackrel{P}{\nrightarrow} 0$, both $S_{n}$ and $T_{n}-1$ are bounded in probability. This lack of precision does not allow these concepts of outlier proneness/resistance to provide a clean classification scheme for distribution functions based on their tail heaviness. Thus, for example, the exponential distribution is absolutely outlier-prone even when it is considered to be a medium-tailed distribution. This issue will be addressed later when concepts for classifying distribution functions are presented. As discussed by Green, ${ }^{16}$ the connection of Definition 3 with the residual life function is as follows:

Theorem 1. Under the assumptions of Definition 3,

(a) is equivalent to $\lim _{x \rightarrow \infty} R_{x}(t)=0$ for all $t>0$.

(b) is equivalent to $\lim _{x \rightarrow \infty} R_{x}(\varepsilon x)=0$ for all $\varepsilon>0$.

(c) is equivalent to $R_{x}(t) \geq \alpha$ for some constants $t, \alpha>0$, and all $x$.

(d) is equivalent to $R_{x}(\varepsilon x) \geq \alpha$ for some constants $\varepsilon, \alpha>0$, and all $x$.

Although not noted by Green, ${ }^{16}$ the following corollary follows easily. ${ }^{25}$

Corollary 1. Under the assumptions of Definition 3,

(1) absolutely outlier-resistant implies relatively outlier-resistant.

(2) relatively outlier-prone implies absolutely outlier-prone.

Green ${ }^{16}$ provided a classification of distributions according to tail behavior through the concepts of outlier-proneness and outlier-resistance. Unfortunately such classification does not provide as clear a picture as it is desirable. A cleaner connection between tail heaviness and the asymptotic behavior of the extreme spacing is possible as discussed further below. The next section considers partial orderings of distributions based on the asymptotic behavior of various quantities.

\section{PARTIAL ORDERS IN TERMS OF TAIL HEAVINESS}

Any attempt to define heavy-tailed distributions needs to first address the question of 'heavy tails compared to what'? Initially, because of the strong influence of the normal distribution on statistical inference, attempts were made to define long-tailedness of a density by taking the normal density as the reference distribution. Thus, for example, Gel et al. ${ }^{26}$ proposed tests of normality against heavy-tailed distributions.

Therefore, using the standard normal as the reference distribution, a density $f(x)$ is heavy-tailed if

$$
\lim _{x \rightarrow \infty} \frac{\phi(x)}{f(x)}=0
$$


where $\phi(x)$ represents the standard normal density. Other definitions replace the normal density in Eq. (2) by the exponential density. Thus, the density $f$ is heavy-tailed if, for all $\lambda>0$,

$$
\lim _{x \rightarrow \infty} \frac{e^{-\lambda x}}{f(x)}=0
$$

Yet another definition classifies the density $f$ as longtailed if it is regularly varying. That is, if for some $\alpha>0$,

$$
f(x)=l(x) x^{-\alpha},
$$

where $l(x)$ is a slowly varying function at infinity, and $\alpha>0$ is the 'tail index'. Recall that a slowly varying function is any function $l$ with $\lim _{x \rightarrow \infty} l(t x) / l(x)=$ 1 , for all $t>0$. Examples of slowly varying functions $l$ are given by $l(x)=(\log (x))^{\alpha}$, for all $\alpha \in R$. Classification of distributions according to tail behavior can then be defined through the criteria (2-4). Thus for example, a density $f$ is short-tailed, compared with the normal density, if the limit of the ratio in Eq. (2), as $x \rightarrow \infty$, is $\infty$. And $f$ is said to be medium-tailed when the limit in Eq. (2) is a finite positive constant. Likewise, definitions for short- and medium tails can be given in terms of the limiting value of the ratio in Eq. (3).

Regarding Eq. (4), the density $f$ can be said to be long-tailed if $\alpha=0$ and short-tailed when $\alpha=\infty$. That is, $f$ is long- or short-tailed depending on whether $f$ is a slowly- or rapidly varying function at infinity.

It is also possible to define partial-tail orders of distributions. The literature of stochastic orders of probability distributions is vast. An important subset of this large class of ordering concepts is the subclass that focuses its attention on ordering distributions according to their tail weight. Although some of these concepts have been labeled tail-orderings, in many important cases these orderings are not puretail orderings in the sense that the comparison of probability distributions depends also on the behavior of the distributions at their centers. Thus, for example, although it is clear that the Cauchy distribution has a heavier tail than the double-exponential distribution, it turns out that the latter is not smaller than the former with respect to the convex 'tail-ordering' of van Zwet. ${ }^{27}$ Loh $^{28}$ discusses several 'tail-orderings' in the case of estimating the location parameter of symmetric distributions. Like the convex ordering, these orderings are sensitive to the behavior of the distribution at its center. Doksum ${ }^{29}$ introduced a tailordering in the context of studying power properties of rank tests. Thus, for distributions $F$ and $G$,
$F \stackrel{t}{<} G$ provided that $F^{-1} G(x)-x$ be nondecreasing, where $F^{-1}$ is the quantile function of $F$. However, it turns out that when $F$ and $G$ are continuous and strictly increasing, this concept is equivalent to the concept of dispersive order introduced by Fraser ${ }^{30}$ and Brown and Tukey, ${ }^{31}$ and hence it is not a tailordering but rather an ordering based on the spread of distributions.

However, pure-tail orderings can be defined along the same lines as those indicated by (2) and (3).

Definition 4. Let $f$ and $g$ define density functions. Then $f$ is said to have a lighter-, equivalent-, or heavier tail than $g$ if $\lim _{x \rightarrow \infty} \frac{f(x)}{g(x)}$ is zero, a constant $0<c<\infty$, or $\infty$, respectively.

Two possible drawbacks of the definition are that the supports of the distributions may be different and then the definition breaks down, and also that densities may not exist. When the densities do not exist, a pure-tail ordering may be given in terms of the ratio of the survival distributions. Rojo ${ }^{2,32}$ provided the following definitions.

Definition 5. Let $F$ and $G$ be probability distribution functions. Then,

$$
\begin{aligned}
& F \leq_{D} G \text { if } \limsup _{x \rightarrow \infty} \bar{F}(x) / \bar{G}(x)<\infty, \\
& F<_{D} G \text { if } F \leq_{D} G \text { but } G{\varliminf_{D}} F, \\
& F \sim_{D} G \text { if } F \leq_{D} G \text { and } G \leq_{D} F .
\end{aligned}
$$

As it turns out, the pure-tail ordering $\leq_{D}$ is not location or scale invariant as indicated by the following example in Ref 2.

Example. Let $f(x)=\phi((x-\mu) / \sigma)$ and $g(x)=\phi((x-$ $\theta) / \tau)$, where $\phi(x)$ denotes the standard normal density and $\mu, \theta$ and $\sigma, \tau$ represent the means and standard deviations respectively. It follows that

$$
\begin{aligned}
\lim _{x \rightarrow \infty} f(x) / g(x) \\
\quad= \begin{cases}0 & \text { if } \tau>\sigma \text { or, if } \tau=\sigma \text { and } \theta \sigma^{2}>\mu \tau^{2} \\
\infty & \text { if } \tau<\sigma \text { or, if } \tau=\sigma \text { and } \theta \sigma^{2}<\mu \tau^{2} \\
1 & \text { otherwise. }\end{cases}
\end{aligned}
$$

It is clear that this lack of invariance applies as well to partial orderings that could be defined with respect to a reference distribution such as those orderings defined, for example, through (2) and (3). As an illustrative example consider (3) and let $f(x) \propto$ $x^{\alpha-1} \mathrm{e}^{-\beta * x}$, for $x>0, \alpha>0, \beta>0$. It follows that the limit in (3) equals 1 when $\lambda=\beta$ and $\alpha=1$; it equals 0 if $\lambda=\beta$ and $\alpha>1$; and it equals $\infty$ if $\lambda=\beta$ and $\alpha<1$. Otherwise the limit in (3) is $\infty$ if $\beta>\lambda$ or 0 if $\beta<\lambda$. 
This lack of invariance, coupled with the fact that the definition for $\leq_{D}$ does not apply when $F$ and $G$ do not have the same support, motivates the following definition.

Definition 6. Let $F$ and $G$ be distribution functions with respective quantile functions given by $F^{-1}$ and $G^{-1}$. Then,

$$
\begin{aligned}
& F \leq_{q} G \text { if } \limsup _{u \rightarrow 1} F^{-1}(u) / G^{-1}(u)<\infty, \\
& F<_{q} G \text { if } F \leq_{q} G \text { but } G \leq_{q} F, \\
& F \sim_{q} G \text { if } F \leq_{q} G \text { and } G \leq_{q} F .
\end{aligned}
$$

Clearly, the $q$-order can order distributions with different, finite or infinite, supports. Moreover, the $q$-order is seen to be location and scale invariant under mild conditions. Rojo ${ }^{33}$ discusses preservation properties of the $\leq_{q}$ and $\leq_{D}$ orders under reliability operations. Thus, both the $\leq_{q}$ and $\leq_{D}$ orders are preserved by convolutions, formation of coherent systems, and finite mixtures. Moreover, under additional mild conditions on the nondecreasing function $h, X \leq_{D} Y\left(X \leq_{q} Y\right)$ implies that $h(X) \leq_{D}$ $h(Y)\left(h(X) \leq_{q} h(Y)\right)$. Rojo ${ }^{34,21}$ examines connections between these orderings and concepts of residual life, and characterizations of these orderings in terms of concepts of aging are discussed.

The following result in Rojo $^{2}$ characterizes these orders. Let $\mathcal{S}=\left\{s:[0,1] \rightarrow \mathcal{R}^{1}\right\}$ be the set of functions with domain $[0,1]$. Henceforth, it will be assumed that all the distribution functions have supports that include $\mathcal{N}_{0}$, a fixed neighborhood of $\infty$. Let

$$
\begin{aligned}
& \mathcal{H}_{0}=\{h: h \text { is nondecreasing and continuous } \\
& \text { in } \left.\mathcal{N}_{0} \text { and } h(x) \rightarrow \infty \text { as } x \rightarrow \infty\right\}, \\
& \mathcal{H}_{1}=\left\{h: h \text { is strictly increasing in } \mathcal{N}_{0} \text {, and } h(s(x))\right. \\
& =O(h(t(x))) \text { as } x \rightarrow 1 \text {, for } s, t \in \mathcal{S} \text {, with } s(x)= \\
& O(t(x)) \text { as } x \rightarrow 1\}, \text { and } \\
& \mathcal{H}_{2}=\left\{h: h \in \mathcal{H}_{1} \text { with } \limsup _{u \rightarrow 1} h(s(u)) / h(t(u))\right. \\
& \left.=\infty, \text { if } \limsup _{u \rightarrow 1} s(u) / t(u)=\infty\right\} .
\end{aligned}
$$

The set of functions $\mathcal{H}_{1}$ includes increasing functions with bounded derivative and, somewhat more generally, functions $h$ with $h(x) \geq x$ and satisfying a Lipschitz condition. For a function $h \in \mathcal{H}_{0}$ or $h \in \mathcal{H}_{1}$, define $b^{*}(x)=\sup \{s: h(s) \leq x\}$.

Theorem 2. Let $X$ and $Y$ be random variables with respective distribution functions given by $F$ and $G$. Then,

(i) $X \leq_{D} Y$ if and only if $h(X) \leq_{D} h(Y)$ for all $h \in \mathcal{H}_{0}$. (ii) $X \leq_{q} Y$ if and only if $h(X) \leq_{q} h(Y)$ for all $h \in \mathcal{H}_{1}$.

(iii) $X<_{q} Y$ if and only if $h(X)<_{q} h(Y)$ for all $h \in \mathcal{H}_{2}$.

Proof: Note that the identity function $h(x)=x$ is in $\mathcal{H}_{0}, \mathcal{H}_{1}$, and $\mathcal{H}_{2}$. Therefore, the only if parts in (i), (ii), and (iii) follow immediately. The if part in (i) follows immediately since for $h \in \mathcal{H}_{0}$, the distribution functions of $h(X)$ and $h(Y)$ are given respectively by $F h^{*}$ and $G b^{*}$. Similarly, for $h \in \mathcal{H}_{1}$ and $h \in \mathcal{H}_{2}$, $b^{*}(h(x))=x$ and then $\left(F b^{*}\right)^{-1}=b F^{-1}$.

Rojo $^{32}$ discussed the relationships between the $<_{q}$ and the $<_{D}$ orders. The comparison between $<_{q}$ and $<_{D}$ can be made more precise when restricting attention to the following classes of distributions.

Definition 7. Let $F$ denote a distribution function.

(i) $F$ is said to have a swiftly varying right tail, $(F \in \mathrm{SVT})$ if there is $t>1$ such that

$$
\liminf _{x \rightarrow \infty} \bar{F}(x) / \bar{F}(t x)>1 .
$$

(ii) $F$ is said to have a scale-invariant right tail, $(F \in \mathrm{SIT})$, if

$$
\limsup _{x \rightarrow \infty} \bar{F}(x) / \bar{F}(2 x)<\infty .
$$

(iii) $F$ is said to have a polynomial tail $(F \in \mathcal{P})$, if $F \in S V T$ and $F \in S I T$.

(iv) $F$ is said to have an exponential tail $(F \in \mathcal{E})$, if for some $t>1$

$$
\begin{aligned}
& \liminf _{x \rightarrow \infty} \frac{\log \bar{F}(t x)}{\log \bar{F}(x)}>1 \text { and } \\
& \limsup _{x \rightarrow \infty} \frac{\log \bar{F}(t x)}{\log \bar{F}(x)}<\infty .
\end{aligned}
$$

It turns out that $\leq_{D}$ and $\leq_{q}$ orderings agree when either $F$ or $G \in \mathcal{P}$. However, when $F$ or $G$ have a slowly varying tail, the approach based on the ratio of the quantile functions provides a more thorough comparison of distribution functions in the sense that $q$-ordering may strongly order the distributions $F$ and $G$, while $D$-ordering may conclude that $F$ and $G$ have equivalent tails. One such example occurs when $\bar{G}(x)=1 / 2 \log (x)$ and $\bar{F}(x)=1 / \log (x)$. When $F$ or $G$ have tail that decrease at an exponential, or faster, rate, then the converse holds. This is the case, for example, when $f$ is the standard normal density, and $g(x)=(1 / 2)|x| \mathrm{e}^{-x^{2} / 2}$. 


\section{CLASSIFICATION OF DISTRIBUTIONS BY LONG-, MEDIUM-, SHORT TAILS}

Pareto $^{35}$ appears to be the first to propose a concept of 'pure-tail' behavior for a probability distribution. Empirical evidence based on income data led Pareto to propose a model for the tail of the income distribution. In his 1897 work, 'The New Theories of Economics', Pareto writes that

Fortunately the figures representing the distribution of wealth group themselves according to a very simple law, which I have been enabled to deduce from unquestioned statistical data. ${ }^{1}$

The 'very simple law' that Pareto referred to is then given as a footnote

This law is as follows: $N=\frac{A}{(x+b)^{\alpha}}$. In which $N$ represents the number of individuals having an income greater than $x$ or $A ; b$ is a constant which for aggregate incomes is in general zero, or very near it; $\alpha$ is another constant whose value lies between 1 and 2 . The law applies only to incomes a little above the minimum. The form of the curve in the immediate neighborhood of this minimum income is still undetermined, for statistics do not furnish us sufficient information for its determination. Since the publication of my Cours I have examined many new statistical data, and they all verify the law which I have formulated. The results of my later investigations have been published in the Giornale degli Economisti Rome.

Note that Pareto admits that the law applies well only in the tail of the income distribution and that the form of the distribution near the minimum is 'still undetermined'. This 'Pareto distribution' has survived in the same form, although several variations of it are also part of the literature. Thus a Pareto distribution can be defined by its density function

$$
f(x)=k x^{-\alpha}, \alpha>1, k>0 .
$$

See for example, Johnson et al. ${ }^{36}$ for other variations of the Pareto distribution. The form given by Eq. (8) can be generalized to include distributions with polynomial tails defined by densities of the form

$$
f(x)=l(x) x^{-\alpha}, \alpha>1,
$$

where, $l(x)$ is a function of slow variation at $\infty$. When $f$ satisfies Eq. (9), $f$ is said to be a function of regular variation at $\infty$. Functions of regular variation have served as models for long - tailed distributions and they play a fundamental role in the theory of extremes. Recall the fundamental theorem of extreme value theory. Let $X_{1}, \ldots, X_{n}$ denote a random sample from the distribution $F$, and let $X_{(1)}, \ldots, X_{(n)}$ denote the order statistics.

Theorem 3. Let $X_{(n)}$ denote the maximum of a sequence of independent and identically distributed, according to $F$, random variables. Suppose that there are sequences of constants $\left\{a_{n}\right\}$ and $\left\{b_{n}\right\}$ such that

$$
a_{n}\left(X_{(n)}-b_{n}\right) \stackrel{\mathcal{D}}{\rightarrow} \mathcal{G},
$$

where $\mathcal{G}$ denotes a nondegenerate distribution. Then, $\mathcal{G}$ must be one of only three possible types:

$$
\begin{aligned}
\Phi_{\alpha}(x) & =\mathrm{e}^{-x^{-\alpha}} I_{\{x>0\}}, \quad \alpha>0, \\
\Psi_{\alpha}(x) & =\mathrm{e}^{-\left(-(x)^{-\alpha}\right)} I_{\{x \leq 0\}}+I_{\{x>0\}}, \quad \alpha>0, \\
\Lambda(x) & =\mathrm{e}^{-\mathrm{e}^{-x}}, \quad x \in \mathcal{R} .
\end{aligned}
$$

The distribution $F$ is then said to be in the domain of attraction of the extreme value Frechet, Weibull, or Gumbel distributions depending on whether Eqs (11), (12), or (13) hold. Although there are distributions $F$ for which sequences $\left\{a_{n}\right\}$ and $\left\{b_{n}\right\}$ cannot be found so that Eq. (10) holds, the conditions for Theorem 3 to hold are relatively mild. And when Eq. (10) holds, it is possible to characterize the distributions $F$ that belong to the domain of attraction of each of the three extreme value distributions. These characterizations take the form of conditions on the tail of $F$. Thus we have the following result. ${ }^{37}$ Let $x_{F}$ denote the right-end point of the support of $F$.

Theorem 4. Let $F$ be a distribution function.

(i) $F$ is in the domain of attraction of $\Phi_{\alpha}$, that is Eq. (10) holds with $\mathcal{G}$ given by Eq. (11), if and only if $\bar{F}(x)$ is regularly varying with exponent $\alpha$.

(ii) $F$ is in the domain of attraction of $\Psi_{\alpha}$, that is Eq. (10) holds with $\mathcal{G}$ given by Eq. (12), if and only if $x_{F}<\infty$, and $\bar{F}\left(x_{F}-x^{-1}\right)$ is regularly varying at $x_{F}$.

(iii) $F$ is in the domain of attraction of $\Lambda$, that is Eq. (10) holds with $\mathcal{G}$ given by Eq. (13), if and only if there is $y<x_{F}$ such that

$$
\bar{F}(x)=c(x) \exp \left\{-\int_{y}^{x} \frac{m(t)}{n(t)} d t\right\}, \quad y<x<x_{F},
$$

where $m(x) \rightarrow 1$ and $c(x) \rightarrow c$ as $x \rightarrow x_{F}$, and $n(x)>$ 0 with $\lim _{x \rightarrow x_{F}} n^{\prime}(x)=0$.

For example, the normal, exponential, and lognormal distributions are in the domain of 
attraction of $\Lambda$. The Pareto, Lomax, and Burr distributions are in the domain of attraction of $\Phi_{\alpha}$, and the uniform distribution on $(0,1)$ as well as the Beta distributions are in the domain of attraction of $\Psi_{\alpha}$. Thus, it is possible to classify distributions according to their tail behavior by using Theorem 4 .

Definition 8. The distribution $F$ is said to have long-, medium-, or short tails, depending on whether $F$ is in the domain of attraction of $\Phi_{\alpha}, \Lambda$, or $\Psi_{\alpha}$ for some $\alpha$.

Some possible drawbacks of the classification proposed by the previous definition are the technical conditions needed for $F$ to be in the domain of attraction of one of the extreme value distributions, together with the fact that, according to the definition, the exponential, the normal, and the lognormal distributions all are classified as medium-tailed. Because the statistical folklore considers the normal as having shorter tails than the exponential, while the lognormal is considered to be long-tailed, the classification based on Definition 8 appears not to have enough sensitivity to classify these distributions into different classes.

An alternative classification scheme due to Parzen $^{22}$ classifies distributions by tail behavior based on the limiting behavior of the density-quantile function.

Definition 9. Let $F$ be a distribution function with right-continuous quantile function $F^{-1}$ and density function $f$. The function $f F^{-1}$ is called the densityquantile function of $F$.

Using results from Andrews, ${ }^{38}$ Parzen $^{22}$ argues that the density quantile for most distributions has the property that

$$
f F^{-1}(u) \sim(1-u)^{\alpha}, \text { as } u \rightarrow 1,
$$

for some $\alpha>0$, where $f F^{-1}(u) \sim(1-u)^{\alpha}$ means that $\lim _{u \rightarrow 1} f F^{-1}(u) /(1-u)^{\alpha}=c$ for some positive constant $c$. When $\alpha=1$, it is possible in many cases to give a more precise representation for the densityquantile function

$$
\begin{aligned}
& f F^{-1}(u) \sim(1-u)(-\log (1-u))^{1-\beta}, \\
& \quad \text { for some } \beta \in[0,1] .
\end{aligned}
$$

The parameters $\alpha$ and $\beta$ are the tail exponent and the shape parameter, respectively. Under some technical conditions-including the twicedifferentiability of the density-quantile function, the tail exponent may be given a precise definition. ${ }^{22}$

A distribution $F$ is classified as long-, medium-, or short-tailed depending on whether $\alpha>1, \alpha=1$, or $\alpha<1$, respectively.
Besides the somewhat harsh technical conditions requiring the twice-differentiability of the densityquantile function, it turns out that Parzen's classification is equivalent to the classification obtained through the extreme value theorem when $F$ can be classified through both approaches. Thus, according to Parzen's classification, the exponential, lognormal, normal, Weibull (i.e., $f(x)=c x^{c-1} \mathrm{e}^{-x^{c}}$ ), and extreme value (i.e., $f(x)=\mathrm{e}^{x} e^{-\mathrm{e}^{x}}$ ) are all medium-tailed distributions. Of course one thinks of the extreme value and the Weibull distribution with $c>1$ as short-tailed distributions while the lognormal and Weibull with $c<1$ as long-tailed distributions.

Although not indicated in Ref 22, it is possible to subclassify the medium-tailed class of distributions by the shape parameter $\beta$ in Eq. (16). A mediumtailed distribution is then medium-medium, mediumshort, medium-long depending on whether $\beta$ is equal to 1 , larger than 1 , or smaller than 1 . Thus, for example, the Weibull distribution with $f(x)=\gamma x^{\gamma-1} \mathrm{e}^{-x^{\gamma}}$, and $f F^{-1}(u)=\gamma(1-u)\{-\log (1-$ $u)\}^{1-1 / \gamma}$, is medium-medium, medium-short, mediumlong depending on whether the $\gamma$ is equal to 1 , larger than 1 , or smaller than 1 . The normal distribution is medium-short as is the extreme value distribution with $f(x)=\mathrm{e}^{x} e^{-\mathrm{e}^{x}}$. However, there are medium-tailed distributions, such as the lognormal, that do not accept the representation given by Eq. (16).

An alternative subclassification of the medium class that agrees with the one in terms of $\beta$ can be given in terms of the limiting value of the failure rate function when this limit exists. Let $r(t)$ be the failure rate function defined by

$$
r(x)=f(x) / \bar{F}(x) .
$$

Lemma 1. (Ref 23) Let $\lim _{x \rightarrow \infty} r(x)$ exist. Then,

(i) $F$ is medium-short if $\alpha=1$ and $\lim _{x \rightarrow \infty}$ $r(x)=\infty$.

(ii) $F$ is medium-medium if $\alpha=1$ and $\lim _{x \rightarrow \infty}$ $r(x)=c$, with $0<c<\infty$.

(iii) $F$ is medium-long if $\alpha=1$ and $\lim _{x \rightarrow \infty}$ $r(x)=0$.

Thus, according to this refinement of Parzen's classification, the lognormal is medium-long, the normal is medium-short, and the exponential and the logistic are medium-medium. Schuster ${ }^{23}$ also made the connection between the asymptotic behavior of the failure rate function of $F$ and that of the Extreme Spacing (ES) from a random sample from $F$ defined by 
$S_{n}=X_{(n)}-X_{(n-1)}$, where $X_{(i)}$ denotes the $i$ th ordered statistic from $F$.

Theorem 5. Let $X_{1}, \ldots, X_{n}$ be a random sample from $F$ with density $f$ and quantile function $F^{-1}$. Define $S_{n}=X_{(n)}-X_{(n-1)}$. Suppose that $\lim _{x \rightarrow \infty} r(x)$ exists and denote this limit by $c$. Then,

(i) $S_{n} \stackrel{p}{\rightarrow} \infty$ if and only if $c=0$.

(ii) $S_{n} \stackrel{p}{\rightarrow} 0$ if and only if $c=\infty$.

(iii) $S_{n}$ is bounded in probability but $S_{n}$ does not converge to 0 in probability if and only if $0<c<\infty$.

It is then possible to classify distributions using Theorem 5. Thus, $F$ is ES-short, ES-long, or ESmedium, according to whether (i), (ii), or (iii) in Theorem 5 holds. Unfortunately, this classification does not agree with the other classifications provided in terms of the tail exponent. Thus, a normal distribution is ES-short but it is medium-short according to the classification in Lemma 1.

Rojo ${ }^{24}$ considered a different approach based on the residual life function. Assuming that $\lim _{t \rightarrow \infty} R_{x}(t)$ exists, for all $t$, it is possible to use this limiting value of $R_{x}(t)$ to classify distributions and then make a more natural and cleaner connection with the asymptotic behavior of the extreme spacing.

Definition 10. Suppose that $\lim _{x \rightarrow \infty} R_{x}(t)$ exists, for all $t$, and denote this limit value by $c$. Then, $F$ is said to have short-, medium-, or long tails if $c$ is 0 , $0<c<\infty$, or $c=\infty$, respectively.

Although the requirement that $\lim _{x \rightarrow \infty} R_{x}(t)$ exists, for all $t$, is a rather mild condition, it is possible for the limit not to exist. See Ref 24 for an example. It is possible to characterize the various classes given by Definition 10 in terms of functions of regular variation. Recall that a function $g(x)$ is rapidly varying at $\infty$ if $\lim _{t \rightarrow \infty} g(t x) / g(x)$ equals 0 or $\infty$ depending on whether $x$ is greater than or less than 1 . Henceforth, $R_{-\infty}, R_{\alpha}$, and $R_{0}$ will denote the class of rapidly varying functions, regularly varying functions with exponent $\alpha$, and slowly varying functions, respectively. We now have the following characterization of the classes given by Definition 10 .

Theorem 6. Suppose $\lim _{x \rightarrow \infty} R_{x}(t)=c$ exists, for all $t$. Then

$$
\begin{aligned}
c=0 & \text { if and only if } \bar{F}(\log x) \in R_{-\infty} \\
c=\infty & \text { if and only if } \bar{F}(\log x) \in R_{0} \\
0<c<\infty & \text { if and only if } \bar{F}(\log x) \in R_{\alpha} \text { for some } \alpha .
\end{aligned}
$$

It turns out that according to this classification, the normal and extreme value distributions are now short-tailed, the Weibull distribution is short-, medium-, or long-tailed depending on whether the shape parameter $\gamma$ is greater than, equal to, or smaller than 1 . This suggests that a classification scheme based on the asymptotic behavior of the residual life function provides a cleaner and more natural approach to classify distributions. Moreover, this classification agrees with the scheme provided by the asymptotic behavior of the extreme spacing $S_{n}$, as indicated in the following theorem, see Rojo ${ }^{24}$ which strengthens Schuster ${ }^{23}$ results.

Theorem 7. Let $F$ be a distribution function with residual life function $R_{x}(t)$ and suppose that $\lim _{t \rightarrow \infty} R_{x}(t)=c$ exists, for all $t$. Let $E$ denote an exponential random variable with scale $\lambda$. Then,

$$
\begin{aligned}
& S_{n} \stackrel{\text { a.s. }}{\rightarrow} 0 \text { if and only if } \bar{F}(\log x) \in R_{-\infty} \\
& S_{n} \stackrel{\text { a.s. }}{\rightarrow} \infty \text { if and only if } \bar{F}(\log x) \in R_{0} \\
& S_{n} \stackrel{\text { a.s. }}{\rightarrow} E \text { if and only if } \bar{F}(\log x) \in R_{\alpha} \text { for some } \alpha .
\end{aligned}
$$

Note that Theorem 7 is stronger than Theorem 5 in at least two counts. First, the convergence is almost sure convergence (a.s.), and also, the class of medium-tailed distributions is completely identified. It follows from Refs 39 and 40, that when $\lim _{x \rightarrow \infty} R_{x}(t)$ exists then the three cases indicated in Theorem 6 are collectively exhaustive. It could then be argued that the correct approach to classifying distributions by tail behavior is provided by Theorem 6 since this scheme agrees more naturally and cleanly, with the classification based on the extreme spacing $S_{n}$, but equally important, it also agrees with the common classification used in the statistical folklore. It must also be noted that the condition that $\lim _{x \rightarrow \infty} R_{x}(t)=0$ for all $t$ has been shown to be necessary and sufficient ${ }^{16}$ for $S_{n} \stackrel{P}{\rightarrow} 0$. Because, as a consequence of Theorems 6 and 7, it can be concluded that $\lim _{x \rightarrow \infty} R_{x}(t)=0$ for all $t$ is equivalent to $S_{n} \stackrel{\text { a.s. }}{\rightarrow} 0$, a clarification is needed. The following argument is a summary of those given in Ref 24 . Note that the condition $\lim _{x \rightarrow \infty} R_{x}(t)=0$ for all $t$ implies that $\bar{F}(\log x) \in R_{-\infty}$. Therefore, the inverse of $\bar{F}(\log x)$ is a slowly varying function $L$ at zero. It follows that $\bar{F}^{-1}(y)=\log L(y)$. Since $\bar{F}^{-1}\left(1-F\left(X_{(n)}\right)\right)=X_{(n)}$, and $\bar{F}^{-1}\left(1-F\left(X_{(n-1)}\right)\right)=X_{(n-1)}$, we can write

$$
S_{n}=\log \left\{\frac{L\left(\bar{F}\left(X_{(n)}\right)\right)}{L\left(\bar{F}\left(X_{(n-1)}\right)\right)}\right\}=\log \left\{\frac{L\left(V * \bar{F}\left(X_{(n-1)}\right)\right)}{L\left(\bar{F}\left(X_{(n-1)}\right)\right),}\right\}
$$


where $V$ is independent of $\bar{F}\left(X_{(n-1)}\right)$. Since $0<V<1$ and $\bar{F}\left(X_{(n-1)}\right) \rightarrow 0$ with probability one, and since $L$ is slowly varying at zero, it follows that $S_{n} \stackrel{\text { a.s. }}{\rightarrow} 0$ as $n \rightarrow \infty$.

\section{CONCLUSION}

Some of the first attempts to classify probability distributions by tail behavior originated with applications in the modeling and analysis of astronomical data and the related issue of whether to eliminate observations which seemed to be far from the rest of the data. In addition, empirical evidence on the distribution of incomes led Pareto to consider long-tailed distributions as models for these types of data. The issue of wealth inequality in the United States has been discussed vigorously in the last few months, with a low percentage of individuals possessing a large percentage of the wealth. Several works have modeled this wealth inequality by various means. We only mention the work by Aaberge et al. ${ }^{41}$ Its complete bibliographical information is given in Further Reading section. However, despite several works that provide methodologies to decide how to deal with outliers, the concept of tail heaviness of a probability distribution and the corresponding classification and ordering schemes based on tail weight remained elusive. This study provided a brief historical treatise of the concept of tail heaviness, concluding that an approach based on the asymptotic behavior of the residual life function and its corresponding characterizations in terms of the extreme spacings and functions of regular variation provides the more natural and cleaner concepts.

The discussion has been restricted to the onedimensional case. The multivariate case offers several challenges that seem difficult to address using the concepts and ideas presented in this review. Nevertheless, approaches based on work of R. Serfling and others using depth-based ideas have been successful in addressing questions connected with multivariate outliers and in the development of robust procedures. Extensions of the concepts of regular variation to the multivariate case to model heavy-tailed distributions have been proposed and connections with the dependence functions of the corresponding copulas have been studied, but results similar to those presented here in the one-dimensional case are presently lacking. See the section on further reading for references on depth-based approaches and multivariate regular variation.

\section{REFERENCES}

1. Wang W, Yatracos Y. A stop-loss risk index. Insur Math Econ 2004, 34:241-250.

2. Rojo J. On the concept of tail-heaviness. Technical Report No. 175. Statistics Department, University of California at Berkeley, 1988.

3. Bernoulli D. Dijudicatio maxime probabilis plurium observationum discrepantium atque verisimillima inductio inde formanda. Acta Academiae Scientiorum Petropolitanae 1777, 1:3-33.

4. Allen CG. The most probable choice between several discrepant observations and the formation therefrom of the most likely induction, Studies in the History of Probability and Statistics: XI. Daniel Bernoulli on Maximum Likelihood. Biometrika 48, 3-13 (English translation of D. Bernoulli’s (1777) work), 1961.

5. Legendre AM. Nouvelles Methods por la Determination des Orbites des Cometes. Paris: Courcier; 1805.

6. Peirce B. Criterion for the rejection of doubtful observations. Astron J 1852, 2:161-163.

7. Airy GB. A letter from Professor Airy, Astronomer Royal, to the Editor. Astron J 1856, 4:137-138.

8. Glaisher JWL. On the rejection of discordant observations. Mon Not R Astron Soc 1872, 33:391-402.
9. Barnett V, Lewis T. Outliers in Statistical Data. 3rd ed. New York: John Wiley and Sons; 1994.

10. Newcomb S. Discussion and Results of Observations on Transits of Mercury from 1677 to 1881. Astron Pap 1882, 1:363-487 (Published by the U.S. Nautical Almanac Office).

11. Newcomb S. A generalized theory of the combination of observations so as to obtain the best result. Am J Math 1886, 8:343-366.

12. Tukey JW. A survey of sampling from contaminated distributions. In: Olkin I, ed. Contributions to Probability and Statistics. Stanford University Press: Palo Alto; 1960, 448-485.

13. Stigler SM. Simon Newcomb, Percy Daniell, and the history of robust estimation 1885-1920. J Am Stat Assoc 1973, 60:872-879.

14. Neyman J, Scott EL. Outlier proneness of phenomena and of related distributions. In: Rustagi J, ed. Optimizing Methods in Statistics. New York: Academic Press; 1971.

15. Green RF. A note on outlier-prone families of distributions. Ann Statist 1974, 2:1293-1295.

16. Green RF. Outlier-prone and outlier-resistant distributions. J Am Stat Assoc 1976, 71:502-505. 
17. Gather U. Über Ausrei $\beta$ ertests und Ausre $\beta$ eranfälligkeit von Wahrscheinlichkeitsverteilungen. Dissertation, RWTH Aachen, 1979.

18. Gather U. Eine charakterisierung des ausrei $\beta$ erverhaltens von verteilungen mit hilfe der dixon-statistik. Methods Oper Res 1981, 44:81-90.

19. Gather U. Tests und Schätzungen in Ausrei $\beta$ ermodellen, Habilitationsschrift, RWTH Aachen. 1984.

20. Mathar R. The outlier behaviour of distributions and the decay of power of optimal tests at decreasing level. Statistics 1989, 20:247-254.

21. Rojo J. Relationships between pure tail orderings of lifetime distributions and some concepts of residual life. Ann Instit Statist Math 1996a, 48:247-255.

22. Parzen E. On tail categorization of probability laws. J Am Stat Assoc 1979, 91:378-384.

23. Schuster EF. Classification of probability laws by tail behavior. J Am Statist Assoc 1984, 79:936-939.

24. Rojo J. On tail categorization of probability laws. J Am Stat Assoc 1996b, 91:378-384.

25. Gather U, Rauhut B. The outlier behaviour of probability distributions. J Stat Plan Infer 1990, 26:237-252.

26. Gel Y, Miao W, Gastwirth JL. Robust directed tests of normality against heavy-tailed alternatives. Comput Stat Data Anal 2007, 51:2734-2746.

27. van Zwet WR. Convex transformations of random variables. CWI Math. Centre Tract 7. Centrum voor Wiakunde en Informatica, Amsterdam, 1964.

28. Loh W-Y. Bounds on are's for restricted classes of distributions defined via tail-orderings. Ann Statist 1984, 12:685-701.

29. Doksum K. Starshaped transformations and the power of rank tests. Ann Math Statist 1969, 40:1167-1176.
30. Fraser DAS. Nonparametric Methods in Statistics. New York: Wiley; 1957.

31. Brown G, Tukey JW. Some distributions of sample means. Ann Math Statist 1946, 7:1-12.

32. Rojo J. A pure-tail ordering based on the ratio of the quantile functions. Ann Statist 1992, 20:570-579.

33. Rojo J. On the preservation of some pure-tail orderings by reliability operations. Statist Probab Lett 1993, 17:189-198.

34. Rojo J. Characterizations of some concepts of aging. IEEE Trans Reliability 1995, 44:285-290.

35. Pareto V. The new theories of economics. J Polit Econ $1897,5: 485-502$.

36. Johnson NL, Kotz S, Balakrishnan N. Continuous Univariate Distributions, 2nd Edition. New York: Wiley and Sons; 1994.

37. Embrechts P, Kluppelberg C, Mikosch T. Modelling Extremal Events. Berlin: Springer Verlag; 1997.

38. Andrews D. A general method for the approximation of tail areas. Ann Statist 1973, 1:367-372.

39. Balkema AA, de Haan L. Residual Life TIme at Great Age. Ann Probab 1974, 2:792-804.

40. Battacharjee MC. Tail Behavior of Age-Smooth Failure Distributions and Applications. Reliability and Quality Control, AP Basu (Ed.) Amsterdam: North-Holland; 1986.

41. Aaberge R, Bjerve S, Doksum K. Modeling inequality and spread in multiple regression. Optimality, The Second Erich L. Lehmann Symposium, J. Rojo, ed. Lecture Notes and Monograph Series, Inst Math Statist, 2006, 49:120-130.

\section{FURTHER READING}

Charpentier A, Segers J. Tails of multivariate archimedean copulas. J Multivariate Anal 2009, 100:1521-1537.

Dang X, Serfling R. Nonparametric depth-based multivariate outlier identifiers, and masking robustness properties. J Stat Plan Infer 2010, 140:198-213.

Kotz S, Nadarajah S. Extreme Value Distributions: Theory and Applications. London: Imperial College Press; 2000.

Donoho DL, Gasko M. Breakdown properties of location estimates based on halfspace depth and projected outlyingness. Ann Statist 1992, 20:1803-1827.

Li H, Sun Y. Tail dependence for heavy-tailed scale mixtures of multivariate distributions. J Appl Probab 2009, 46:925-937.

Liu RY. Data depth and multivariate rank tests. In: Dodge Y, ed. L1-Statistics and Related Methods. Amsterdam: North-Holland; 1992, 279-294.

Liu RY, Parelius JM, Singh K. Multivariate analysis by data depth: descriptive statistics, graphics and inference. Ann Statist 1999, 27:783-858.

Serfling R. Nonparametric multivariate descriptive measures based on spatial quantiles. J Stat Plan Infer 2004, 123:259-278.

Serfling R. Depth functions in nonparametric multivariate analysis. In: Liu RY, Serfling R, Souvaine DL, eds. Data Depth: Robust Multivariate Analysis, Computational Geometry and Applications. Providence, RI: American Mathematical Society; 2006, 1-16. 
Serfling R. Equivariance and invariance properties of multivariate quantile and related functions, and the role of standardization. J Nonparametr Stat 2010, 22:915-936.

Weng C, Zhang Y. Characterization of multivariate heavy-tailed distribution families via copula. J Multivariate Anal 2012, 106:178-186.

Zuo Y, Serfling R. General notions of statistical depth function. Ann Statist 2000, 28:461-482.

Zuo Y, Serfling R. On the performance of some nonparametric location measures relative to a general notion of multivariate symmetry. J Stat Plan Infer 2000, 84:55-79. 